Article

\title{
Free and Nanoencapsulated Tobramycin: Effects on Planktonic and Biofilm Forms of Pseudomonas
}

\author{
Eulalia Sans-Serramitjana ${ }^{1}$, Marta Jorba ${ }^{1}$, Ester Fusté ${ }^{1,2}$, José Luis Pedraz ${ }^{3}$, Teresa Vinuesa ${ }^{1}$ \\ and Miguel Viñas ${ }^{1, *}$ \\ 1 Laboratory of Molecular Microbiology and Antimicrobials, Department of Pathology and Experimental \\ Therapeutics, Faculty of Medicine \& Health Sciences, University of Barcelona, 08007 Barcelona, Spain; \\ eulalia.bio.87@gmail.com (E.S.-S.); m.jorba.pedrosa@gmail.com (M.J.); \\ esterfustedominguez@ub.edu (E.F.); tvinuesa@ub.edu (T.V.) \\ 2 School of Nursing, Faculty of Medicine \& Health Sciences, University of Barcelona, 08007 Barcelona, Spain \\ 3 Laboratory of Pharmaceuticals, University of the Basque Country and Biomedical Research Networking \\ Center in Bioengineering, Biomaterials and Nanomedicine (CIBER-BBN), 48940 Lejona, Spain; \\ joseluis.pedraz@ehu.es \\ * Correspondence: mvinyas@ub.edu; Tel.: +34-934-024-265
}

Received: 16 April 2017; Accepted: 19 June 2017; Published: 26 June 2017

\begin{abstract}
Cystic fibrosis (CF) is a genetic disorder in which frequent pulmonary infections develop secondarily. One of the major pulmonary pathogens colonizing the respiratory tract of CF patients and causing chronic airway infections is Pseudomonas aeruginosa. Although tobramycin was initially effective against $P$. aeruginosa, tobramycin-resistant strains have emerged. Among the strategies for overcoming resistance to tobramycin and other antibiotics is encapsulation of the drugs in nanoparticles. In this study, we explored the antimicrobial activity of nanoencapsulated tobramycin, both in solid lipid nanoparticles (SLN) and in nanostructured lipid carriers (NLC), against clinical isolates of $P$. aeruginosa obtained from CF patients. We also investigated the efficacy of these formulations in biofilm eradication. In both experiments, the activities of SLN and NLC were compared with that of free tobramycin. The susceptibility of planktonic bacteria was determined using the broth microdilution method and by plotting bacterial growth. The minimal biofilm eradication concentration (MBEC) was determined to assess the efficacy of the different tobramycin formulations against biofilms. The activity of tobramycin-loaded SLN was less than that of either tobramycin-loaded NLC or free tobramycin. The minimum inhibitory concentration (MIC) and MBEC of nanoencapsulated tobramycin were slightly lower (1-2 logs) than the corresponding values of the free drug when determined in tobramycin-susceptible isolates. However, in tobramycin-resistant strains, the MIC and MBEC did not differ between either encapsulated form and free tobramycin. Our results demonstrate the efficacy of nanoencapsulated formulations in killing susceptible P. aeruginosa from $\mathrm{CF}$ and from other patients.
\end{abstract}

Keywords: tobramycin; lipid nanoparticles; antibacterial and antibiofilm effects; P. aeruginosa; cystic fibrosis

\section{Introduction}

Cystic fibrosis (CF) is the most common genetic disorder in the Caucasian population and it is characterized by a high morbidity and mortality. The CF lung is compromised by the production of viscous mucus secretions, resulting in a debilitated mucociliary clearance that promotes bacterial infection and inflammation [1]. Pseudomonas aeruginosa is the predominant opportunistic pathogen infecting the respiratory tract of CF patients. Once chronic lung colonization occurs, P. aeruginosa changes phenotypically to produce alginate, which allows the bacterium to become established within 
mucoid biofilms and thus highly resistant to multiple antimicrobials [2,3]. Indeed, the emergence of multidrug-resistant phenotypes and treatment failure were shown to correlate with the reduced permeability of the outer membrane of P. aeruginosa to most antimicrobials and the acquisition of genes, encoding antimicrobial resistance $[4,5]$.

Tobramycin is a hydrophilic, cationic antibiotic administered as an aerosol in the treatment of P. aeruginosa lung infections in CF patients [6]. Like other aminoglycosides, tobramycin targets the bacterial ribosome, such that bacterial resistance, although rare, mainly involves impermeability and the acquisition of aminoglycoside-modifying enzymes, encoded either on a plasmid or within the genome by transposable elements [7]. However-despite their chemical stability, fast bactericidal effect, synergy with B-lactam antibiotics, and low incidence of resistance-aminoglycosides are of limited utility because of their nephrotoxicity [8,9]. While tobramycin is less nephrotoxic than gentamicin and other aminoglycosides and has been successfully used against P. aeruginosa [10], planktonic bacteria are much more sensitive than bacteria in biofilms, the growth form occurring in the lower respiratory tract of CF patients. The reduced efficacy of tobramycin and other antimicrobials is due to poor mucus penetration, the resilience of the extracellular matrix of the biofilm, and inactivation of the drug through various binding interactions in the infected CF lung [11]. The mechanisms of tobramycin resistance are, to our knowledge, not fully understood. The relationship between mucoidity and tobramycin resistance has been explored; the main conclusion is that mucoidity per se has no effect on resistance [12]. However, the study also distinguished between the roles of mucoidity and biofilm formation in the ability of $P$. aeruginosa to resist antibiotic treatment. While biofilm formation expectantly increased resistance of PAO1 to tobramycin, uncontrolled alginate production did not. Thus, one should speculate that other mechanisms than external matrix have to be involved in the resistance caused by biofilm. It has been shown that iron regulation clearly affects susceptibility, but also gene expression differences.

Nanoformulations such as lipid nanoparticles could improve the delivery of tobramycin and thus enhance its activity. Lipid nanoparticles with a solid matrix are available as solid lipid nanoparticles (SLN) and as newer-generation lipid nanostructured lipid carriers (NLC). SLN are composed of solid lipids. NLC are prepared from a blend of a solid lipid with a liquid lipid. Both are stabilized by surfactants and are able to incorporate lipophilic and hydrophilic drugs [13].

Among the key benefits of lipid nanoparticles in the pulmonary delivery of antibiotics are the improved bioavailability and rapid distribution of the drug; precise targeting of the site of infection; the need for a lower dose; longer administration intervals, thereby reducing the risk of serious dose-related side effects; and the scaling-up feasibility of nanoparticle production [14]. The specific advantages of SLN and NLC over other delivery systems include their higher stability compared to liposomes, both in vitro and in vivo [13,15], as well as their better biocompatibilities and lower potential toxicity (both acute and chronic) compared to polymeric nanoparticles and other synthetic formulations [15,16]. Moreover, the use of nanoparticles could overcome pre-existing drug resistance mechanisms, including those involving the decreased uptake and increased efflux of the drug, to achieve better biofilm penetration. Previous studies testing the effectiveness of aminoglycosides against clinical isolates of $P$. aeruginosa reported better results with compounds loaded in nanoformulations than with the free drug [17-19].

Based on these findings and our own results demonstrating the antimicrobial activity of colistin loaded into lipid nanoparticles [20,21], in this work we explored the activity of nanoencapsulated (both SLN and NLC) tobramycin versus that of the free drug against P. aeruginosa clinical isolates obtained from CF patients. We then investigated the efficacy of these novel formulations in the eradication of $P$. aeruginosa biofilms. The main purpose was to demonstrate that after their inclusion in nanoparticles tobramycin was able to conserve its antimicrobial activity. Even when antimicrobial action is not higher, the nanoparticles are of interest since pharmacology has demonstrated a better distribution in the respiratory tree of molecules in lipid nanoparticles. Moreover, we have reported that in vivo 
nanoparticles spread homogenously through the lung and there is no migration of lipid nanoparticles to other organs, such as liver, spleen, or kidneys [21].

\section{Materials and Methods}

\subsection{Bacterial Isolates}

The 34 clinical isolates of P. aeruginosa (17 non-mucoid and 17 mucoid) included in this study were obtained from the sputum samples and pharyngeal exudates of CF patients seen at the University Hospital Vall d'Hebrón and University Hospital Sant Joan de Déu (Barcelona, Spain) between January and April 2012. The patients (59\% female, $41 \%$ male) ranged in age from 9 to 50 years (mean: 27 years). P. aeruginosa strains ATCC 27853 and PAO1 served as the control strains in the drug susceptibility assays and biofilm studies, respectively. Two CF clinical isolates, $P$. aeruginosa strain $362 \mathrm{VH}$ (tobramycin-resistant) and strain 056SJD (tobramycin-susceptible), were used to evaluate the ability of free and nanoencapsulated tobramycin to inhibit bacterial growth and eradicate bacterial biofilms. Table 1 summarizes the isolates used and their main characteristics.

Table 1. Bacterial strains used in this research. Strains SJD were isolated in Sant Joan de Déu Hospital and those being VH in the Hospital of Vall d'Hebrón. Abbreviations: Piper/Tz: Piperacillin/Tazobactam; Caz: Ceftazidime; Azt: Aztreonam; Imp: Imipenem; Mero: Meropenem; Gnt: Gentamicin; Tobra: Tobramycin; Amk: Amikacin; Col: Colistin; Cpfx: Ciprofloxacin. S: Susceptible; R: Resistant; I: Intermediate.

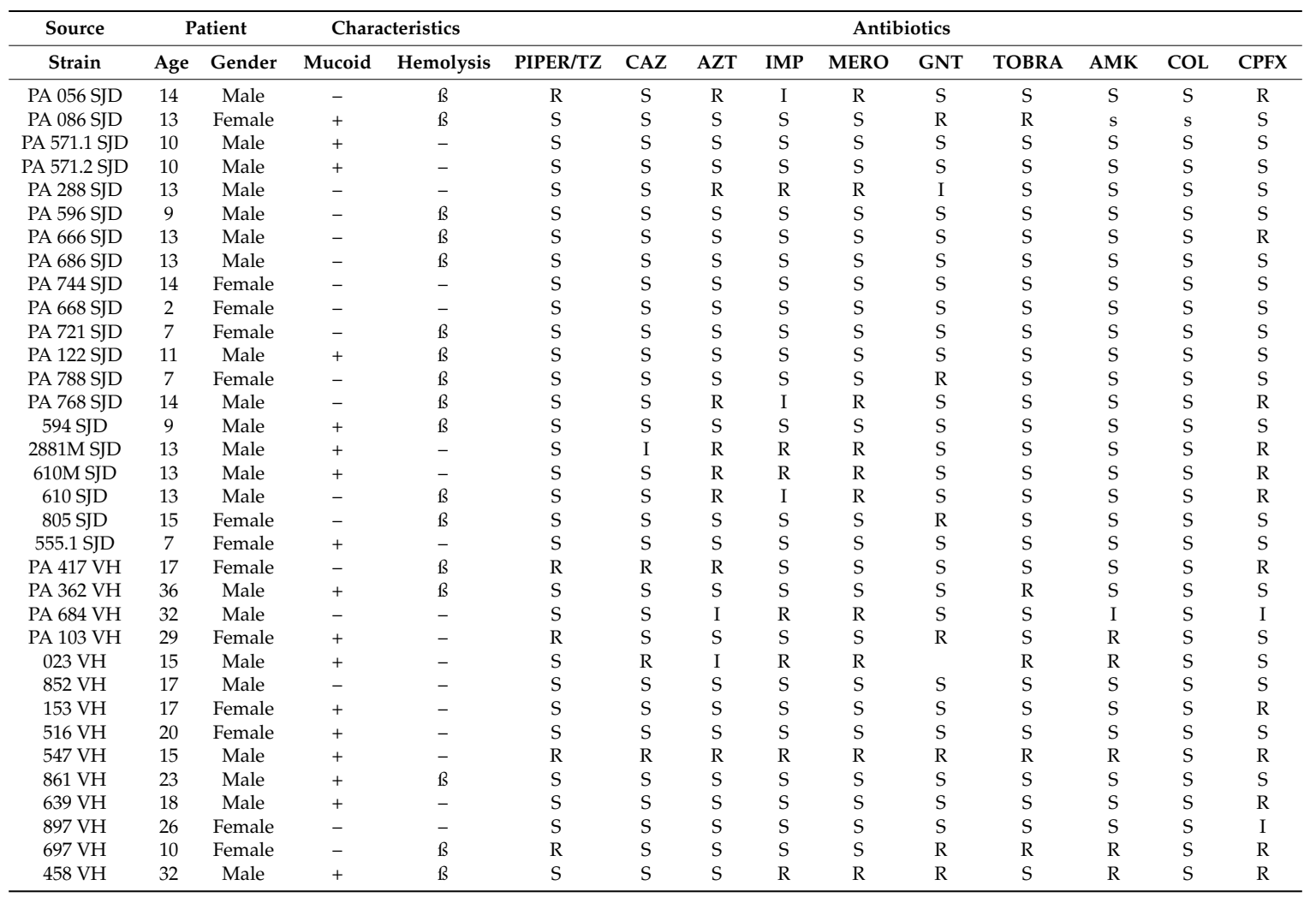




\subsection{Chemicals and Bacteriological Media}

Tobramycin was purchased from Sigma-Aldrich Chemicals (St. Louis, MO, USA). Mueller-Hinton II broth cation-adjusted (MHBCA) was from Becton Dickinson (Sparks, MD, USA). Tryptone soy agar (TSA) was purchased from Sharlau (Sentmenat, Barcelona, Spain). Precirol ATO 5 was kindly provided by Gattefossé (Madrid, Spain), and poloxamer 188 by BASF (Ludwigshafen, Rhineland-Palatinate, Germany). Polysorbate and Tween 80 were purchased from Panreac Química (Castellar del Vallès, Barcelona, Spain). Miglyol 812 was provided by Sasol (Hamburg, Germany).

\subsection{Preparation of Lipid Nanoparticles}

Tobramycin-loaded nanoparticles were prepared as described. Briefly, two loaded formulations were elaborated, namely solid lipid nanoparticles (SLN) and nanostructured lipid carriers (NLC) [21]. An emulsion solvent evaporation technique was chosen for the preparation of SLN. Briefly, $10 \mathrm{mg}$ of antibiotic (Sigma-Aldrich, St. Louis, MO, USA) were mixed with a $5 \%(w / v)$ Precirol ${ }^{\circledR}$ ATO 5 (Gattefossé, Madrid, Spain) dichloromethane solution. Then, the organic phase and an aqueous surfactant containing solution (Poloxamer 188 at $1 \% w / v$ and Polysorbate 80 at $1 \% w / v$ ) were mixed and emulsified by sonication at $20 \mathrm{~W}$ for $30 \mathrm{~s}$ (Branson Sonifier 250, Danbury, CT, USA). The solvent was allowed to evaporate by magnetic stirring for $2 \mathrm{~h}$ at room temperature. Subsequently, the resulting SLNs were washed by centrifugation in Amicon ${ }^{\circledR}$ centrifugal filtration units $(100,000$ MWCO, Merck Millipore, Billerica, MA, USA) at $2500 \mathrm{rpm}$ for $15 \mathrm{~min}$ three times. For the NLC elaboration, a hot melt homogenization technique was selected. In brief, Precirol ${ }^{\circledR}$ ATO 5 and Miglyol ${ }^{\circledR} 812$ (Sasol, Johannesburg, South Africa) were selected as the lipid core. Those lipids were mixed with the API and heated above the melting temperature of the solid lipid. The surfactant solution consisted of $1.3 \%(w / v)$ of Polysorbate 80 and $0.6 \%(w / v)$ of Poloxamer 188. The lipid and aqueous solutions were heated to the same temperature and then emulsified by sonication for $15 \mathrm{~s}$ at $20 \mathrm{~W}$. Nanoparticles were stored at $4{ }^{\circ} \mathrm{C}$ overnight to allow lipid re-crystallization and particle formation. Then, a washing step was undergone by centrifugation at $2500 \mathrm{rpm}$ in Amicon ${ }^{\circledR}$ centrifugal filtration units $(100,000$ MWCO) three times. All the nanoparticles prepared were freeze-dried with two different cryoprotectants, either D-mannitol or trehalose (15\%). In SLN formulations, emulsifiers constituted the aqueous phase of the emulsions, stabilizing the lipid dispersion of the nanoparticles and preventing their agglomeration [22]. Thus, the influence of the emulsifier on the bioactivity of the lipid nanoparticles was examined in two different types of SLN. SLN-tobramycin nanoparticles were prepared using the emulsifiers poloxamer 188 and polysorbate 80 , each at $1 \% w / v$. SLN-SDS-tobramycin nanoparticles were prepared using $2 \%$ sodium dodecyl sulfate (SDS) as the co-emulsifier. NLCs loaded with tobramycin (NLC-tobramycin) were prepared using a hot melt homogenization technique, following the method described by Pastor et al. [21].

All three types of nanoparticles used in this work (SLN-tobramycin, SLN-SDS-tobramycin, and NLC-tobramycin) were stabilized by trehalose, since in previous research we determined that it was a better cryoprotectant than mannitol [20]. Solid Lipid Nanoparticles and Nanostructured lipid carriers were characterized for size, polidispersity index (PDI) and Z-potential by means of Zetaseiser Nano ZS (Malvern Instruments, Worcestershire, UK). Measurements were based on Dynamic Light Scattering (DLS). Atomic force microscopy images were obtained by using a XE-70 atomic force microscope (Park Systems, Suwon, Korea).

\subsection{Drug Susceptibility Assay in Planktonic Bacteria}

Susceptibility to free tobramycin and to the three formulations of nanoencapsulated tobramycin was determined using the broth microdilution method in accordance with the Clinical Laboratory Standards Institute [23]. Briefly, the isolates were grown overnight at $37^{\circ} \mathrm{C}$ in $\mathrm{MHBCA}$, after which $2 \mathrm{~mL}$ of the culture was used to inoculate $20 \mathrm{~mL}$ of fresh MHBCA medium. After $2 \mathrm{~h}$ at $37^{\circ} \mathrm{C}$ and $200 \mathrm{rpm}$, the bacterial cultures were adjusted to an optical density at $625 \mathrm{~nm}\left(\mathrm{OD}_{625 \mathrm{~nm}}\right)$ of $0.08-0.1$ 
and diluted 1:1000 in fresh MHBCA medium. Five $\mu \mathrm{L}$ of each diluted suspension was added to the wells (10 ${ }^{4}$ UFC/well) of 96-well microtiter plates previously filled with MHBCA and serially diluted antibiotic (free and nanoencapsulated). The plates were incubated at $37^{\circ} \mathrm{C}$ for $24 \mathrm{~h}$, after which the minimal inhibitory concentration (MIC) was determined macroscopically, based on the visually assessed turbidity of the wells. All experiments were performed in triplicate with three technical replicates.

\subsection{Effect of Free and Nanoencapsulated Tobramycin on P. aeruginosa Growth}

Two P. aeruginosa CF isolates, tobramycin-susceptible strain 056SJD and tobramycin-resistant strain $362 \mathrm{VH}$, were used to examine the effect of free and nanoencapsulated (SLN and NLC) tobramycin. The antimicrobials were added to exponentially growing liquid cultures $\left(1 \times 10^{8} \mathrm{CFU} / \mathrm{mL}\right.$, in MHBCA) at concentrations above and below the MIC. Samples were taken aseptically at $0,1,2,3,4$, and $5 \mathrm{~h}$ from bacterial cultures incubated at $37^{\circ} \mathrm{C}$ with shaking $(250 \mathrm{rpm})$. Bacterial growth was measured optically to determine the $\mathrm{OD}_{625 \mathrm{~nm}}$. All measurements were carried out in triplicate.

\subsection{Antimicrobial Susceptibility of Sessile Bacteria}

The minimal biofilm eradication concentration (MBEC), defined in this study as the minimal antibiotic concentration required to eliminated $>90 \%$ of the non-treated biofilm, was determined as described by Moskowitz et al. [24], with modifications. Briefly, the formation of bacterial biofilms was promoted as follows: the pegs of a modified polystyrene microtiter lid (catalog No. 445497; Nunc TSP system) were immersed into 96-well microtiter plates containing inoculated (10 4 UFC/well) $200 \mu \mathrm{L}$ MHBCA/well. The modified plates were left undisturbed at $37{ }^{\circ} \mathrm{C}$ for $24 \mathrm{~h}$. The pegs were then gently rinsed in $0.9 \% \mathrm{NaCl}$ and the bacterial biofilms exposed to different concentrations of free and nanoencapsulated tobramycin for $24 \mathrm{~h}$ at $37^{\circ} \mathrm{C}$ in MHBCA. The pegs were then rinsed again with $0.9 \% \mathrm{NaCl}$ and the biofilms removed by $10 \mathrm{~min}$ sonication and centrifugation (2000 rpm, $10 \mathrm{~min})$ in a BioSan Laboratory Centrifuge LMC-3000. Bacteria recovered from the biofilms were incubated for $24 \mathrm{~h}$ at $37^{\circ} \mathrm{C}$. Pegs were again rinsed with $0.9 \% \mathrm{NaCl}$ solution and biofilms removed by $10 \mathrm{~min}$ sonication. Recovered bacteria were incubated for $24 \mathrm{~h}$ at $37^{\circ} \mathrm{C}$. Optical densities at $620 \mathrm{~nm}$ were measured in order to determine MBEC values. All experiments were performed in triplicate on at least three occasions.

\subsection{Statistical Analysis}

The antimicrobial susceptibilities of the tested P. aeruginosa strains to free and nanoencapsulated tobramycin were statistically analyzed using Cochran's $Q$ test. A $p$-value $<0.05$ was considered to indicate statistical significance.

\section{Results and Discussion}

\subsection{Nanoparticle Characterization}

Main characterization data of nanoparticles are shown in Table 2. AFM imaging and size measurements of particles are presented in Figure 1.

Table 2. Characteristics of nanoparticle (TB tobramycin).

\begin{tabular}{ccccc}
\hline Formulation & Mean Size $(\mathbf{n m})$ & PDI & $\begin{array}{c}\text { Zeta-Potential } \\
\mathbf{( m V )}\end{array}$ & $\begin{array}{c}\text { Percentage EE } \\
\text { (Encapsulation Efficiency) }\end{array}$ \\
\hline TB-SLN & $302 \pm 20.5$ & $0.361 \pm 0.02$ & $-20.5 \pm 6.09$ & ND \\
TB-NLC & $254.05 \pm 14.5$ & $0.311 \pm 0.01$ & $-23.03 \pm 2.76$ & $93.15 \pm 0.65$ \\
\hline
\end{tabular}




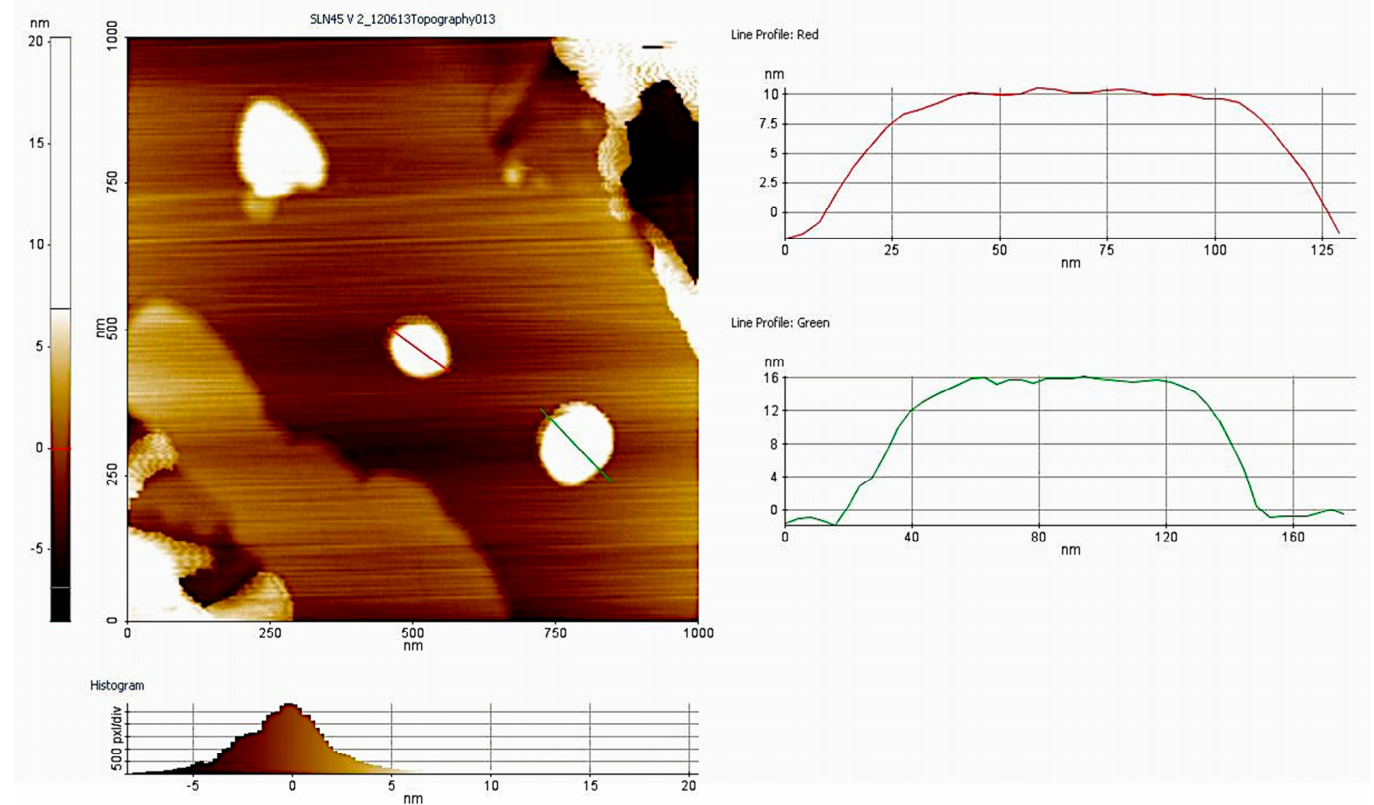

Figure 1. Size measurement performed by AFM imaging. Diameter was around $150 \mathrm{~nm}$.

\subsection{Antimicrobial Activity of Free and Nanoencapsulated Tobramycin}

Nearly all the isolates tested in this study resulted to be susceptible (MIC $\leq 4 \mu \mathrm{g} / \mathrm{mL}$ ) to both the free and nanoencapsulated tobramycin formulations (Figure 1). The MIC of free tobramycin tested against the isolates was $0.5 \mu \mathrm{g} / \mathrm{mL}$, whereas that of NLC-tobramycin was slightly lower (between $0.25 \mu \mathrm{g} / \mathrm{mL}$ and $0.5 \mu \mathrm{g} / \mathrm{mL}$ ) and was also lower than the MICs of SLN-and SLN-SDS-tobramycin (between 1 and $4 \mu \mathrm{g} / \mathrm{mL}$ and $0.5 \mu \mathrm{g} / \mathrm{mL}$, respectively) type.

In addition, NLCs were much more active than either of the SLN preparations, as evidenced by MIC values of 0.5 and $1-4 \mu \mathrm{g} / \mathrm{mL}(p<0.05)$, respectively. Among the two types of SLN, the formulation prepared without SDS lost antimicrobial activity (up to eight-fold higher MICs) (Figure 1). Thus, further experiments were conducted using NLC and SLN-SDS.

The efficient antibacterial activity of lipid nanoparticles loaded with tobramycin may be due to their small size and physic-chemical properties, which facilitates diffusion of the drug into the bacterial cell [25]. Similar results were reported by Ghaffari et al. [19] in their study of $P$. aeruginosa clinical isolates obtained from CF patients. The authors showed that tobramycin loaded in lipid nanoparticles had the same or higher antimicrobial activity than the free form of the drug. The slightly higher bioactivity of tobramycin-loaded NLC than SLN can be attributed to the higher drug-loading capacity of these nanoparticles and the avoidance of drug loss during storage [14,26]. As demonstrated by Moreno-Sastre et al. [27], second-generation NLC are more stable than first-generation SLN and they can be stored at a wider range of temperatures without relevant modifications of their antimicrobial activity.

The improved antibacterial activity of SLN-SDS vs. the SLN particles suggests that SDS, when used as a co-emulsifier, confers improved drug stability and release. SDS may also facilitate contact between the lipid nanoparticles and water, resulting in a better distribution equilibrium of the drug. Of relevance to our findings is the major challenge posed by ensuring drug stability in the development of colloidal drug carriers, which offer a high surface area and short diffusion pathways [28]. Figure $2 \mathrm{~b}$ shows the data separated for mucoid and non-mucoid strains of P. aeruginosa. 
(a)
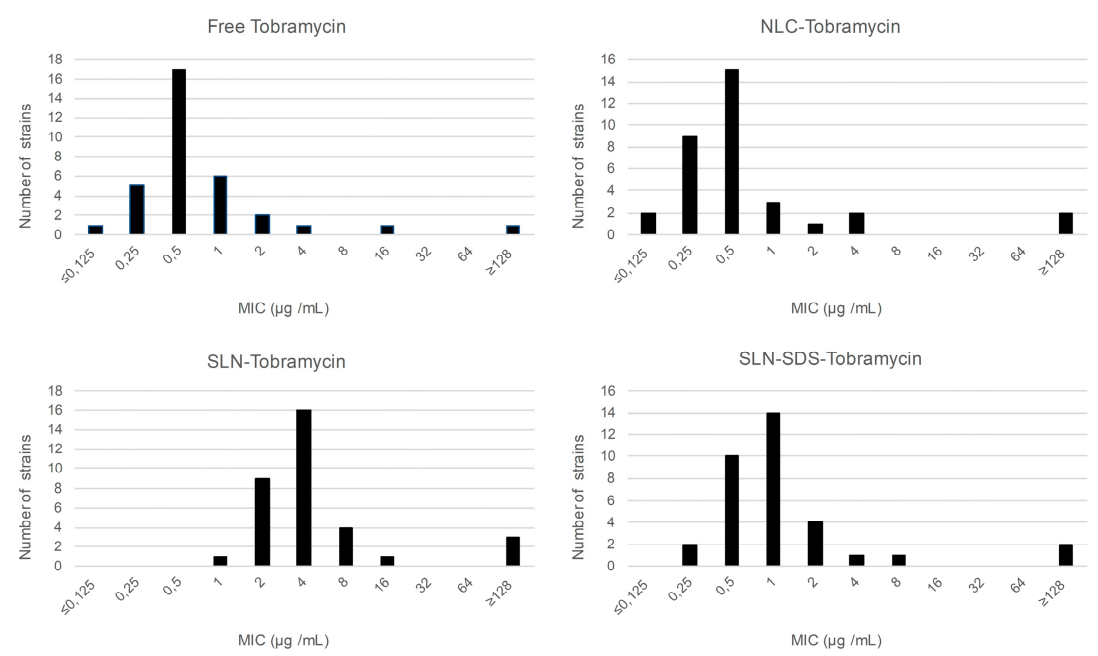

(b)
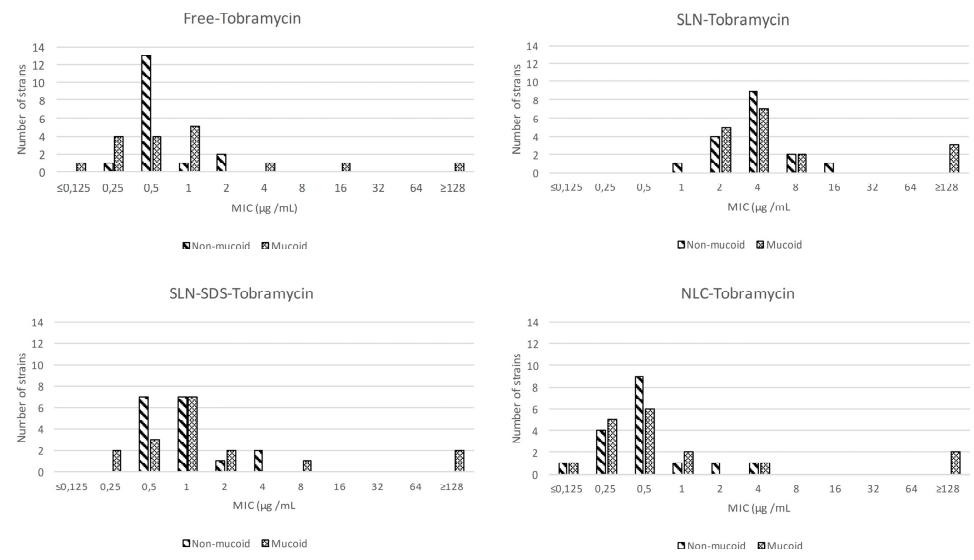

Figure 2. (a) The bioactivity (minimum inhibitory concentration, MIC) of lipid nanoparticles loaded with tobramycin in 34 strains of Pseudomonas aeruginosa isolated from the clinical samples of cystic fibrosis patients; (b) The same as in (a) but separated according to the 17 mucoid and 17 non-mucoid strains of the bacterium. For an explanation of the nanoparticles, see the text.

\subsection{Effect of Free and Nanoaencapsulated Tobramycin on Bacterial Growth}

The susceptibilities of non-mucoid, susceptible (isolate 056SJD) and mucoid, resistant (isolate $362 \mathrm{VH})$ P. aeruginosa to free and nanoencapsulated tobramycin were similar at all concentrations of the antibiotic tested (Figure 3). At sub-inhibitory concentrations $(1 / 2 \times \mathrm{MIC})$, the effect of the tobramycin-loaded lipid formulations on the growth kinetics of susceptible isolate was slightly lower than that of the free drug (Figure 3a) whereas the response of the resistant isolate did not differ (Figure 3d). At the MIC, greater inhibition of the susceptible isolate was achieved, since after $5 \mathrm{~h}$ of antimicrobial exposure none of the formulations was able to fully inhibit the growth of the resistant isolate (Figure $3 \mathrm{~b}, \mathrm{e}$ ). At concentrations above the MIC, the growth of the susceptible isolate was inhibited immediately after the addition of the antimicrobial (Figure 3c), but, again, none of the 
formulations fully inhibited the growth of the resistant isolate (Figure 3f). Empty lipid nanoparticles had no antibacterial activity in either isolate (data not shown).

Taken together, our results demonstrate that the loading of tobramycin into lipid nanoparticles does not adversely affect the antimicrobial activity of the drug against planktonic P. aeruginosa. The preserved potency of lipid nanoparticles containing tobramycin may be due to their facilitated diffusion across the bacterial cell membranes. Mugabe et al. [29] showed that the effective antimicrobial activity of gentamicin loaded into liposomes involved fusion of the particles with the bacterial membrane, leading to its deformation. Further experiments are needed to better understand the interactions between the lipids in nanoformulations and the cellular membrane of microorganisms that promote drug diffusion.

The slower killing of the mucoid, resistant strain of $P$. aeruginosa than of the non-mucoid, susceptible strain by free as well as nanoencapsulated tobramycin can be explained by the additional time needed for outer membrane permeabilization by the drug, regardless of its method of preparation, and the subsequent delay in its reaching its intracellular target.

A previous study showed an immediate effect of tobramycin against most of the susceptible populations tested but not against the resistant population [30].
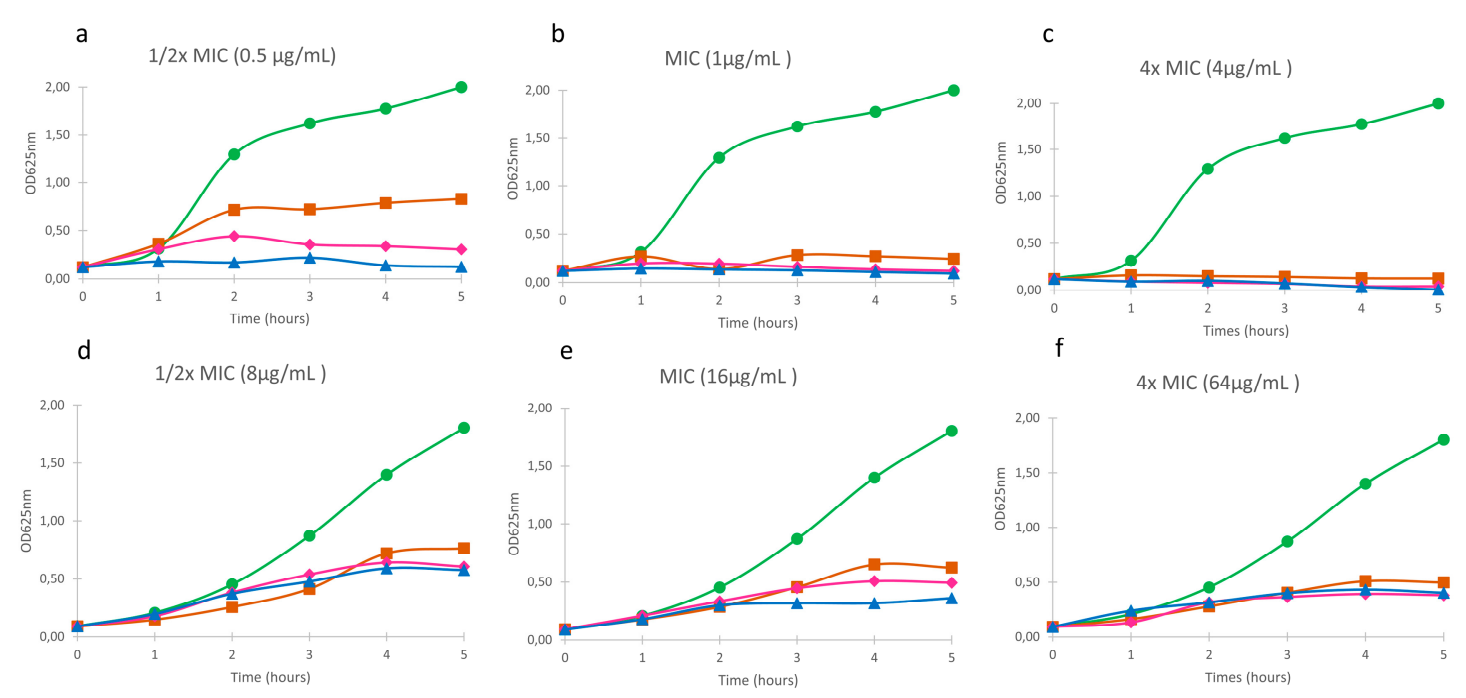

Figure 3. The effect of free and nanoencapsulated (SLN and NLC) tobramycin on the growth of $P$. aeruginosa. (a,b,c) strain 056SJD; (d,e,f) strain 362VH. Control ( $\rightarrow-)$; Free tobramycin $(--)$; SLN-Tobramycin $(\multimap)$; NLC-Tobramycin $(\multimap)$.

\subsection{Anti-Biofilm Efficacy of Free and Nanoencapsulated Tobramycin}

To test the influence of the lipid nanoparticles on tobramycin's ability to kill sessile bacteria, biofilms of four P. aeruginosa strains were exposed to free and nanoencapsulated (SLN and NLC) tobramycin at antibiotic concentrations between 0 and $256 \mu \mathrm{g} / \mathrm{mL}$. ATCC strain 27,853 and strain PAO1 were used as controls, and strains 056SJD (non-mucoid, tobramycin-susceptible) and 362VH (mucoid, tobramycin-resistant) as the $P$. aeruginosa $\mathrm{CF}$ isolates. All $P$. aeruginosa strains used in this experiment formed adequate biofilms (data not shown). The MIC and MBEC values of the four strains are shown in Table 3. Among the isolates susceptible to tobramycin, the MIC and MBEC values of the nanoencapsulated drug were slightly lower (1-2 logs) than those of the free drug. However, for the clinical isolate resistant to tobramycin, there were no differences in the MIC and MBEC values obtained with the nanoparticles and free tobramycin. The exception was NLC-tobramycin, in which the MBEC was slightly lower than the value obtained with the free form. The much higher MBEC vs. MIC values of both free and nanoencapsulated tobramycin likely reflected the interaction between the anionic mucopolysaccharide of the biofilm and the cationic aminoglycoside, 
such that the amount of free tobramycin available to act against the resident bacteria was limited [31]. Among the two types of nanoparticles (SLN and NLC), NLC were slightly more active than SLN $(1 \mathrm{log})$ for all strains tested. Specifically, the concentrations of free tobramycin needed to completely eradicate the $P$. aeruginosa biofilm were $8-16 \mu \mathrm{g} / \mathrm{mL}$ (tobramycin-susceptible strains) and $32 \mu \mathrm{g} / \mathrm{mL}$ (tobramycin-resistant strain), but the effective NLC-tobramycin concentration was lower $(2-4 \mu \mathrm{g} / \mathrm{mL}$ and $16 \mu \mathrm{g} / \mathrm{mL}$, respectively). The better results obtained with the NLC formulation of tobramycin were in agreement with our previously published results showing that colistin-loaded NLCs were highly effective in biofilm eradication [20]. A modification of MBEC assay was performed to test the efficacy of NLC-tobramycin to prevent the biofilm formation [32]. For all the isolates tested, BPC (biofilm prevention concentration) values of NLC-tobramycin were identical to values for free tobramycin. Thus, whereas NLC-tobramycin was more effective than its free form in eradicating biofilms, both free and nanoencapsulated tobramycin did not show any differences on the prevention on biofilm formation.

Table 3. Minimal biofilm eradication concentration (MBEC) and minimum inhibitory concentration (MIC) of free and NLC-encapsulated tobramycin. P. aeruginosa ATCC 27853 and strain PAO1 were used as controls. Strains 056SJD (non-mucoid, tobramycin-susceptible) and 362VH (mucoid, tobramycin-resistant) served as the Pseudomonas.

\begin{tabular}{ccccccccc}
\hline & \multicolumn{2}{c}{ ATCC 27853 } & \multicolumn{2}{c}{ PAO1 } & \multicolumn{2}{c}{ 056SJD } & \multicolumn{2}{c}{ 362VH } \\
\cline { 2 - 8 } & $\begin{array}{c}\text { MIC } \\
(\mu \mathrm{g} / \mathrm{mL})\end{array}$ & $\begin{array}{c}\text { MBEC } \\
(\mu \mathrm{g} / \mathrm{mL})\end{array}$ & $\begin{array}{c}\text { MIC } \\
(\mu \mathrm{g} / \mathrm{mL})\end{array}$ & $\begin{array}{c}\text { MBEC } \\
(\mu \mathrm{g} / \mathrm{mL})\end{array}$ & $\begin{array}{c}\text { MIC } \\
(\mu \mathrm{g} / \mathrm{mL})\end{array}$ & $\begin{array}{c}\text { MBEC } \\
(\mu \mathrm{g} / \mathrm{mL})\end{array}$ & $\begin{array}{c}\text { MIC } \\
(\mu \mathrm{g} / \mathrm{mL})\end{array}$ & $\begin{array}{c}\text { MBEC } \\
(\mu \mathrm{g} / \mathrm{mL})\end{array}$ \\
\hline $\begin{array}{c}\text { Free } \\
\text { Tobramicin } \\
\text { SLN-SDS- }\end{array}$ & 0.5 & 8 & 0.5 & 16 & 1 & 16 & 16 & 32 \\
$\begin{array}{c}\text { Tobramicin } \\
\text { NLC- }\end{array}$ & 0.25 & 4 & 0.25 & 8 & 0.5 & 8 & 16 & 32 \\
Tobramicin & $\leq 0.0625$ & 2 & 0.25 & 4 & 0.25 & 4 & 16 & 16 \\
\hline
\end{tabular}

Although the mechanisms underlying the efficacy of tobramycin in NLC are not fully understood, a role for charge distribution seems likely. Tobramycin loaded into NLC has a negative net charge because of the negatively charged nanoparticles, in contrast to the positive net charge of free tobramycin. The superior mucus penetration of negatively charged nanoparticles has been reported [33] and suggests the greater ability of NLC-tobramycin to penetrate the exopolyssacharide matrix surrounding the biofilm structure. Increased penetration would better allow tobramycin to reach its cellular target, in contrast to its free form. Alternatively, the fast-antimicrobial release reported by Pastor et al. [21] would ensure an initial antimicrobial concentration that is high enough to inhibit the biofilm growth of $P$. aeruginosa. Moreover, a sustained antimicrobial concentration higher than the MIC value would enable the eradication of surviving cells. However, further experiments are needed to determine which, if any, of our hypotheses is the correct one.

\section{Conclusions}

New antimicrobial formulations, such as lipid nanoparticles, can improve the transfer of antimicrobials to their sites of action, potentially allowing a dose reduction and therefore the avoidance of adverse side effects. Our study of planktonic cultures and biofilms of $P$. aeruginosa demonstrated that antimicrobial activity of tobramycin was not affected by nanoencapsulation. Thus, we found that nanoencapsulation of tobramycin did not improve its efficacy against planktonic P. aeruginosa. However, nanoencapsulation did improve its ability to eradicate P. aeruginosa biofilms. Given the key role of biofilms in respiratory infections of P. aeruginosa in CF patients, the results obtained in this study, and especially with NLC-tobramycin, may provide new options in the treatment of these infections, particularly taking into account the better distribution of antibiotics when inhaled as nanoparticles. 
Acknowledgments: The bacterial strains used in this study were generously provided by the Hospital Vall d'Hebron and Hospital Sant Joan de Déu. This work was carried out under the Comprehensive Research on Effective Therapies for the Treatment of Cystic Fibrosis and Associated Diseases (TERFIQEC); IPT-2011-1402-900000 funded by the Spanish Ministry of Economy and Competitiveness. The authors gratefully acknowledge the support of University of the Basque Country UPV/EHU (UFI11/32) and the support of the Basque Government by IT 428-10 consolidated group. MV is member of the ENABLE (European Gram Negative Antibacterial Engine) European consortium (IMI-ND4BB, http:/ /www.imi.europa.eu/content/enable).

Author Contributions: E.S.-S., M.J., and E.F. did the experiments; P.J.L. prepared the nanoformulations, M.V. and T.V. conceived the research and did the interpretation of data; E.S.-S. and M.V. wrote the paper.

Conflicts of Interest: The authors declare no conflict of interest

\section{References}

1. Lyczak, J.B.; Cannon, C.L.; Pier, G.B. Lung infections associated with cystic fibrosis. Clin. Microbiol. Rev. 2002, 15, 194-222. [CrossRef] [PubMed]

2. Wei, Q.; Ma, L.Z. Biofilm matrix and its regulation in Pseudomonas aeruginosa. Int. J. Mol. Sci. 2013, 14, 20983-21005. [PubMed]

3. Drenkard, E.; Ausubel, F.M. Pseudomonas biofilm formation and antibiotic resistance are linked to phenotypic variation. Nature 2002, 416, 740-743. [PubMed]

4. Li, X.Z.; Plésiat, P.; Nikaido, H. The challenge of efflux-mediated antibiotic resistance in Gram-negative bacteria. Clin. Microbiol. Rev. 2015, 28, 337-418. [CrossRef] [PubMed]

5. Fusté, E.; López-Jiménez, L.; Segura, C.; Gainza, E.; Vinuesa, T.; Viñas, M. Carbapenem-resistance mechanisms of multidrug-resistant Pseudomonas aeruginosa. J. Med. Microbiol. 2013, 62, 1317-1325. [PubMed]

6. Döring, G.; Flume, P.; Heijerman, H.; Elborn, J.S. Treatment of lung infection in patients with cystic fibrosis: Current and future strategies. J. Cyst. Fibros. 2012, 11, 461-479. [CrossRef] [PubMed]

7. MacLeod, D.L.; Nelson, L.E.; Shawar, R.M.; Lin, B.B.; Lockwood, L.G.; Dirk, J.E.; Miller, G.H.; Burns, J.L.; Garber, R.L. Aminoglycoside-resistance mechanisms for cystic fibrosis Pseudomonas aeruginosa isolates are unchanged by long-term, intermittent, inhaled tobramycin treatment. J. Infect. Dis. 2000, 181, 1180-1184. [PubMed]

8. Stehling, F.; Büscher, R.; Grosse-Onnebrink, J.; Hoyer, P.F.; Mellies, U. Glomerular and tubular renal function after repeated once-daily tobramycin courses in cystic fibrosis patients. Pulm. Med. 2017, 2017, 1-6.

9. Wargo, K.A.; Edwards, J.D. Aminoglycoside-Induced Nephrotoxicity. J. Pharm. Pract. 2014, $27,573-577$. [CrossRef] [PubMed]

10. Weintraub, R.G.; Duggin, G.G.; Horvath, J.S.; Tiller, D.J. Comparative nephrotoxicity of two aminoglycosides: Gentamicin and tobramycin. Med. J. Aust. 1982, 2, 129-132. [PubMed]

11. Cao, B.; Christophersen, L.; Kolpen, M.; Jensen, P.Ø.; Sneppen, K.; Høiby, N.; Moser, C.; Sams, T. Diffusion retardation by binding of tobramycin in an alginate biofilm model. PLoS ONE 2016, 11, e0153616. [CrossRef] [PubMed]

12. Oglesby-Sherrouse, A.G.; Djapgne, L.; Nguyen, A.T.; Vasil, A.I.; Vasil, M.L. The complex interplay of iron, biofilm formation, and mucoidy affecting antimicrobial resistance of Pseudomonas aeruginosa. Pathog. Dis. 2014, 70, 307-320. [CrossRef] [PubMed]

13. Das, S.; Chaudhury, A. Recent advances in lipid nanoparticle formulations with solid matrix for oral drug delivery. AAPS Pharm. Sci. Tech. 2011, 12, 62-76. [CrossRef] [PubMed]

14. Weber, S.; Zimmer, A.; Pardeike, J. Solid Lipid Nanoparticles (SLN) and Nanostructured Lipid Carriers (NLC) for pulmonary application: A review of the state of the art. Eur. J. Pharm. Biopharm. 2014, 86, 7-22. [CrossRef] [PubMed]

15. Puri, A.; Loomis, K.; Smith, B.; Lee, J.H.; Yavlovich, A.; Heldman, E.; Blumenthal, R. Lipid-based nanoparticles as pharmaceutical drug carriers: From concepts to clinic. Crit. Rev. Ther. Drug Carr. Syst. 2009, 26, 523-580. [CrossRef]

16. Hwang, T.L.; Aljuffali, I.A.; Lin, C.F.; Chang, Y.T.; Fang, J.Y. Cationic additives in nanosystems activate cytotoxicity and inflammatory response of human neutrophils: Lipid nanoparticles versus polymeric nanoparticles. Int. J. Nanomed. 2015, 10, 371-385. 
17. Abdelghany, S.M.; Quinn, D.J.; Ingram, R.J.; Gilmore, B.F.; Donnelly, R.F.; Taggart, C.C.; Scott, C.J. Gentamicin-loaded nanoparticles show improved antimicrobial effects towards Pseudomonas aeruginosa infection. Int. J. Nanomed. 2012, 7, 4053-4063.

18. Deacon, J.; Abdelghany, S.M.; Quinn, D.J.; Schmid, D.; Megaw, J.; Donnelly, R.F.; Jones, D.S.; Kissenpfennig, A.; Elborn, J.S.; Gilmore, B.F.; et al. Antimicrobial efficacy of tobramycin polymeric nanoparticles for Pseudomonas aeruginosa infections in cystic fibrosis: Formulation, characterisation and functionalisation with dornase alfa (DNase). J. Control Release 2015, 198, 55-61. [CrossRef] [PubMed]

19. Ghaffari, S.; Varshosaz, J.; Saadat, A.; Atyabi, F. Stability and antimicrobial effect of amikacin-loaded solid lipid nanoparticles. Int. J. Nanomed. 2011, 6, 35-43.

20. Sans-Serramitjana, E.; Fusté, E.; Martínez-Garriga, B.; Merlos, A.; Pastor, M.; Pedraz, J.L.; Esquisabel, A.; Bachiller, D.; Vinuesa, T.; Viñas, M. Killing effect of nanoencapsulated colistin sulfate on Pseudomonas aeruginosa from cystic fibrosis patients. J. Cyst. Fibros. 2016, 15, 611-618. [CrossRef] [PubMed]

21. Pastor, M.; Moreno-sastre, M.; Esquisabel, A.; Sans, E.; Viñas, M.; Bachiller, D.; Asensio, V.J.; Pozo, A.D.; Gainza, E.; Pedraz, J.L. Sodium colistimethate loaded lipid nanocarriers for the treatment of Pseudomonas aeruginosa infections associated with cystic fibrosis. Int. J. Pharm. 2014, 477, 485-494. [CrossRef] [PubMed]

22. Attama, A.; Momoh, M.A.; Builders, P.F. Lipid Nanoparticulate Drug Delivery Systems: A Revolution in Dosage Form Design and Development. Recent Adv. Nov. Drug Carr. Syst. 2012, 107-140. [CrossRef]

23. Clinical and Laboratory Standards Institute. Performance Standards for Antimicrobial Susceptibility Testing; 27th Informational Supplement CLSI Document M100-S27; Clinical and Laboratory Standards Institute: Wayne, PA, USA, 2017.

24. Moskowitz, S.M.; Foster, J.M.; Emerson, J.; Burns, J.L. Clinically Feasible Biofilm Susceptibility Assay for Isolates of Pseudomonas aeruginosa from Patients with Cystic Fibrosis. J. Clin. Microbiol. 2004, 42, 1915-1922. [CrossRef] [PubMed]

25. De Jong, W.H.; Borm, P.J. Drug delivery and nanoparticles:Applications and hazards. Int. J. Nanomed. 2008, 3, 133-149. [CrossRef]

26. Martins, S.; Sarmento, B.; Ferreira, D.C.; Souto, E.B. Lipid-based colloidal carriers for peptide and protein delivery-Liposomes versus lipid nanoparticles. Int. J. Nanomed. 2007, 2, 595-607.

27. Moreno-Sastre, M.; Pastor, M.; Esquisabel, A.; Sans, E.; Viñas, M.; Bachiller, D.; Pedraz, J.L. Stability study of sodium colistimethate-loaded lipid nanoparticles. J. Microencapsul. 2016, 33, 636-645. [CrossRef] [PubMed]

28. Yadav, N.; Khatak, S.; Vir, U.; Sara, S. Solid lipid nanoparticles-A review. Int. J. Appl. Pharm. 2013, 5, 8-18.

29. Mugabe, C.; Azghani, A.O.; Omri, A. Liposome-mediated gentamicin delivery: Development and activity against resistant strains of Pseudomonas aeruginosa isolated from cystic fibrosis patients. J. Antimicrob. Chemother. 2005, 55, 269-271. [CrossRef] [PubMed]

30. Bulitta, J.B.; Ly, N.S.; Landersdorfer, C.B.; Wanigaratne, N.A.; Velkov, T.; Yadav, R.; Oliver, A.; Martin, L.; Shin, B.S.; Forrest, A.; et al. Two mechanisms of killing of Pseudomonas aeruginosa by tobramycin assessed at multiple inocula via mechanism-based modeling. Antimicrob. Agents Chemother. 2015, 59, 2315-2327. [CrossRef] [PubMed]

31. Khan, W.; Bernier, S.P.; Kuchma, S.L.; Hammond, J.H.; Hasan, F.; O'Toole, G.A. Aminoglycoside resistance of Pseudomonas aeruginosa biofilms modulated by extracellular polysaccharide. Int. Microbiol. 2010, 13, 207-212. [PubMed]

32. Fernández-Olmos, A.; García-Castillo, M.; Maiz, L.; Lamas, A.; Baquero, F.; Cantón, R. In vitro prevention of Pseudomonas aeruginosa early biofilm formation with antibiotics used in cystic fibrosis patients. Int. J. Antimicrob. Agents 2012, 40, 173-176. [CrossRef] [PubMed]

33. Forier, K.; Messiaen, A.S.; Raemdonck, K.; Deschout, H.; Rejman, J.; De Baets, F.; Nelis, H.; De Smedt, S.C.; Demeester, J.; Coenye, T.; et al. Transport of nanoparticles in cystic fibrosis sputum and bacterial biofilms by single-particle tracking microscopy. Nanomedicine 2013, 8, 935-949. [CrossRef] [PubMed]

(C) 2017 by the authors. Licensee MDPI, Basel, Switzerland. This article is an open access article distributed under the terms and conditions of the Creative Commons Attribution (CC BY) license (http:/ / creativecommons.org/licenses/by/4.0/). 